\title{
Single-Carrier FDMA with Blanking/Clipping for Mitigating Impulsive Noise Over PLC Channels
}

\author{
Khaled M. Rabie and Emad Alsusa \\ School of Electrical and Electronic Engineering, \\ The University of Manchester, United Kingdom, M13 9PL, \\ Emails: \{khaled.rabie@manchester.ac.uk; e.alsusa@manchester.ac.uk\}
}

\begin{abstract}
Communication signals over power-line channels can be affected greatly by impulsive noise (IN). The effect of this noise is commonly reduced with the application of a nonlinear preprocessor at the receiver such as blanking, clipping or hybrid (combined blanking and clipping) that blanks and/or clips the received signal when it exceeds a certain threshold. Erroneous blanking/clipping of the unaffected signals can lead to significant performance degradations. It is found that determining the optimal blanking/clipping threshold is the key for achieving best performance. In contract to these studies, we show in this paper that the performance of the nonlinear preprocessing-based method is not only impacted by the blanking/clipping threshold but also by the transmitted signal's peak-to-average power ratio (PAPR). In light of this and for more efficient IN cancellation we, therefore, propose to implement single-carrier FDMA (SCFDMA), which inherently has low PAPR properties, combined with a nonlinear preprocessor at the receiver. The results reveal that the proposed system can provide significant enhancements in terms of minimizing the probability of IN detection error as well as achieving up to $4 \mathrm{~dB}$ gain in the output signal-to-noise ratio relative to the conventional OFDM case.
\end{abstract}

Index Terms-Impulsive noise, interleaved FDMA (IFDMA), localized FDMA (LFDMA), power-line communications (PLC), single-carrier frequency division multiple access (SC-FDMA).

\section{INTRODUCTION}

$\mathbf{T}$ HE main advantage of power-line communications (PLC) is the fact that they utilize a pre-installed infrastructure of wiring networks which can be easily accessed through electricity outlets in the home. Such technology becomes even more attractive in harsh wireless environments where propagation loss is high such as in underground structures and buildings with metal walls [1]. However, the power-line (PL) channel does not represent a favorable channel for communication signals since these cables have never been designed for signal transmission at high frequency. For reliable communications over PLs, it is important to overcome few obstacles including the strong branching problem [2], crosstalk between the wires, noise [3] and high levels of frequency-dependent attenuation [4]. In contrast to many other conventional communication channels, noise over PLs can not be represented as additive white Gaussian noise (AWGN). In fact, it is categorized into background noise and impulsive noise (IN) [3], [5]. The latter is, however, the most dominant factor responsible for degrading communication signals [6]. IN has random occurrence rate with high power spectral density (PSD) and a short duration, [3], [7], but frequently exceeding the signal symbol length which can seriously affect high speed communications [8]. To evaluate the system performance in the presence of IN, the two-component
mixture-Gaussian noise model, [9], [10], has been adopted in this paper.

Several methods have been proposed to minimize the effect of IN in PL orthogonal frequency-division multiplexing (OFDM)-based systems. The simplest of which is to precede the OFDM demodulator with a nonlinear device, such as blanking, clipping or hybrid (combined blanking and clipping) to blank or/and clip the incoming signal when it exceeds certain thresholds [11]. In these methods, the selection of blanking or/and clipping threshold(s) is the key for achieving best performance. Theoretical performance analysis is considered in [10], [11] where closed-form expressions for the signal-tonoise ratios (SNRs) at the output of the nonlinear devices were derived. These studies assume that the IN characteristics, in the form of signal-to-impulsive noise ratio (SINR) and the IN probability of occurrence, can be accurately estimated at the receiver in order to optimally blank IN. Such assumptions, however, may be difficult to fulfill in practice because of the dynamic nature of the PLC channel. In such scenario, estimation errors of noise parameters could lead to imperfect recognition of the IN signal. Consequently, this will cause blanking/clipping uncorrupted signal samples causing IN detection errors and hence dramatic performance deterioration [12]. Not only that, but also uncorrupted signal samples with high amplitude may wrongly trigger the blanker/clipper causing errors [13]. This can be the case in OFDM systems as they suffer from high peak-toaverage power ratio (PAPR). Therefore, in this study we show that the performance of blanking/clipping-based IN mitigation techniques is sensitive not only to the threshold value(s) but also to the signal's PAPR. In contrast to other studies, in this paper we propose to implement single-carrier frequencydivision multiple access (SC-FDMA), which inherently has low PAPR [14], to enhance the overall performance of the system. Two SC-FDMA schemes are considered in this work namely, Localized FDMA (LFDMA) and Interleaved FDMA (IFDMA).

The contribution of this paper is as follows. First we investigate the PAPR of the OFDMA, LFDMA and IFDMA systems and then show how such signals' peaks can affect the blanking/clipping threshold. Two different quantitative characterization aspects of the achievable performance are considered, namely, the probability of IN detection error and the SNR at the output of the blanking, clipping and hybrid devices. The results reveal that SC-FDMA with nonlinear preprocessors at the receiver can considerably minimize the probability of IN detection error and is able to provide up to $4 \mathrm{~dB}$ SNR enhancement relative to the conventional OFDMA system. 


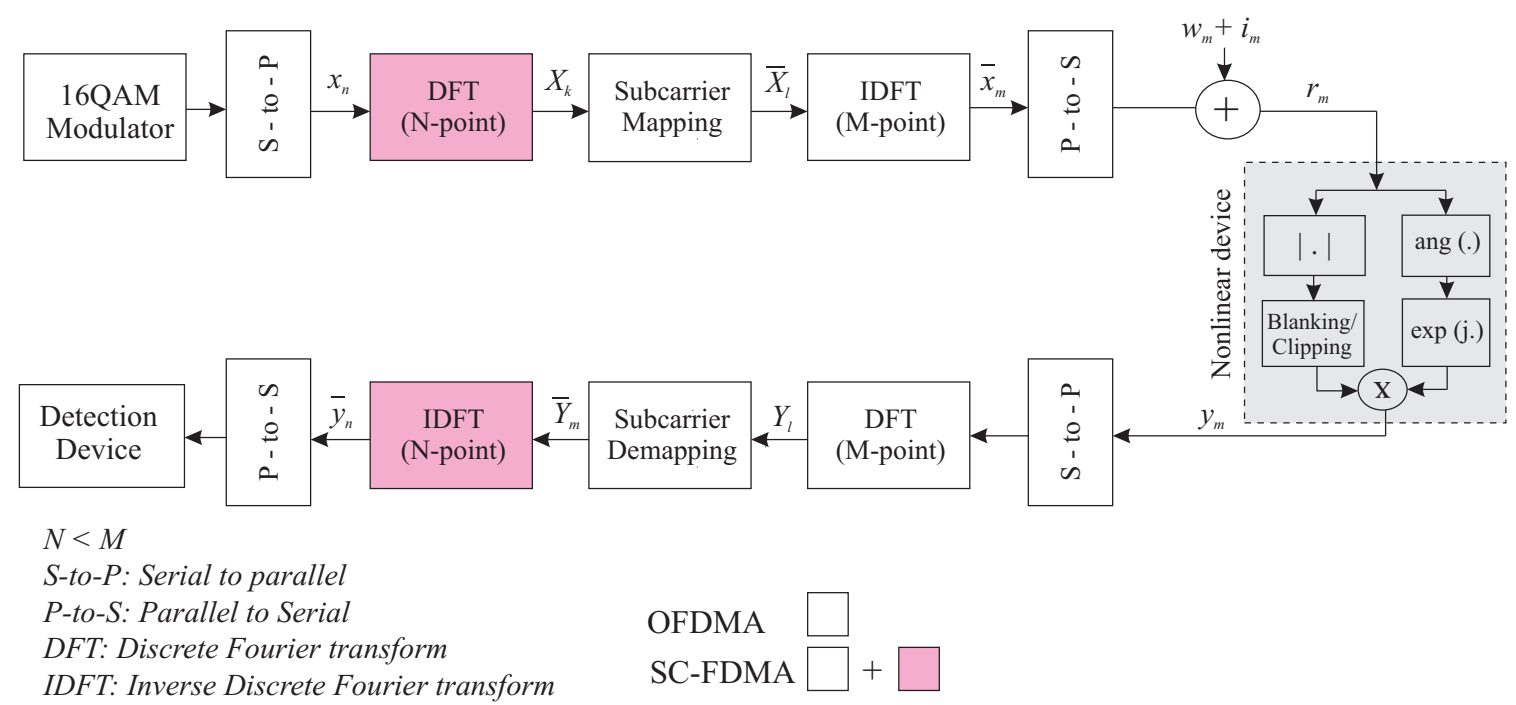

Fig. 1: SC-FDMA system with a nonlinear preprocessor at the receiver.

The rest of this paper is organized as follows. In Section II the system model is described. Section III demonstrates the PAPR performance for the OFDMA, LFDMA and IFDMA systems as well as their relationship with the blanking/clipping threshold. In Section IV, the probability of IN detection error for the OFDMA, LFDMA and IFDMA systems is investigated and the corresponding output SNR performance is presented in Section V. Finally, conclusions are drawn in Section VI.

\section{System Model Overview}

The basic system model used in this study is shown in Fig. 1. First, the information bits are mapped into baseband 16QAM symbols which are then grouped into blocks each of length $N$ symbols by the serial to parallel (S-to-P) converter, $\left\{x_{n}: n=0,1, \ldots, N-1\right\}$. After that, these blocks are passed through an $N$-point discrete Fourier transform (DFT) modulator to produce the frequency domain representation, $\left\{X_{k}: k=0,1, \ldots N-1\right\}$ which is defined as

$$
X_{k}=\sum_{n=0}^{N-1} x_{n} e^{\frac{-j 2 \pi n k}{N}}
$$

$X_{k}$ is then fed to the subcarrier mapping device to produce $\left\{\bar{X}_{l}: l=0,1, \ldots, M-1\right\}$ where $M>N$. Several mapping patterns have been introduced in the literature such as LFDMA and the IFDMA [14]. The principle of these mapping techniques are presented in Fig. 2 for $M=16$ and $N=4$; the ratio $Q=N / M$ denotes the band spreading factor. In the first scheme, consecutive subcarriers are occupied by the DFT outputs with zeros occupying the remaining subcarriers such that

$$
\bar{X}_{l}= \begin{cases}X_{l}, & 0 \leq l \leq N-1 \\ 0, & N \leq l \leq M-1\end{cases}
$$

Whereas in the IFDMA scheme, the DFT outputs are allocated over the entire bandwidth with equal distance while zero padding the unused subcarriers

$$
\bar{X}_{l}= \begin{cases}X_{l / Q}, & l=Q . k \quad(0 \leq k \leq N-1) \\ 0, & \text { Otherwise }\end{cases}
$$

The frequency domain samples $\bar{X}_{l}$ are then passed through an $M$-point inverse discrete Fourier transform (IDFT) modulator to produce $\bar{x}_{m}$, given by (4), before going into the parallelto-serial (P-to-S) convertor and then transmission.

$$
\bar{x}_{m}=\frac{1}{M} \sum_{l=0}^{M-1} \bar{X}_{l} e^{\frac{j 2 \pi m l}{M}}
$$

Using the notation in (4), the PAPR of the transmitted signal can be expressed as

$$
\text { PAPR }=10 \log _{10}\left(\frac{\max _{m=0,1, \ldots, M-1}\left|\bar{x}_{m}\right|^{2}}{\frac{1}{M} \sum_{m=0}^{M-1}\left|\bar{x}_{m}\right|^{2}}\right)
$$

This paper adopts the two-component mixture-Gaussian noise model in which IN is modeled as a Bernoulli-Gaussian random process [9] and is given as

$$
n_{m}=w_{m}+i_{m}, \quad m=0,1,2, \ldots, M-1
$$

where

$$
i_{m}=b_{m} g_{m}, \quad m=0,1,2, \ldots, M-1
$$

$n_{m}$ is the total noise component, $w_{m}$ is the additive white Gaussian noise (AWGN), $i_{m}$ is the IN, $g_{m}$ is complex white Gaussian noise with mean zero and $b_{m}$ is the Bernoulli process with probability mass function 


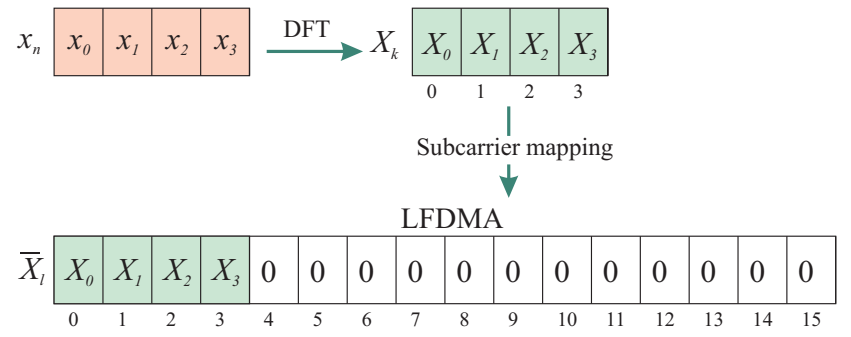

$\bar{X}_{l}$\begin{tabular}{|c|c|c|c|c|c|c|c|c|c|c|c|c|c|c|c|}
\hline$X_{0}$ & 0 & 0 & 0 & $X_{I}$ & 0 & 0 & 0 & $X_{2}$ & 0 & 0 & 0 & $X_{3}$ & 0 & 0 & 0 \\
\hline 0 & 1 & 2 & 3 & 4 & 5 & 6 & 7 & 8 & 9 & 10 & 11 & 12 & 13 & 14 & 15
\end{tabular}

Fig. 2: The frequency domain symbols for LDFMA and IFDMA subcarrier mapping schemes when $N=4, Q=4$ and $M=16$.

$$
\operatorname{Pr}\left(b_{m}\right)=\left\{\begin{array}{ll}
p, & b_{m}=1 \\
0, & b_{m}=0
\end{array} \quad m=0,1, \ldots, M-1\right.
$$

where $p$ is referred to as the IN probability of occurrence. The probability density function (PDF) of the total noise can be expressed as

$$
P_{n_{m}}\left(n_{m}\right)=(1-p) \mathcal{G}\left(n_{m}, 0, \sigma_{w}^{2}\right)+p \mathcal{G}\left(n_{m}, 0, \sigma_{w}^{2}+\sigma_{i}^{2}\right)
$$

$\mathcal{G}($.$) is the Gaussian PDF and is given by \mathcal{G}\left(s, \mu, \sigma_{s}^{2}\right)=$ $\frac{1}{\sqrt{2 \pi \sigma_{s}^{2}}} e^{-\frac{(s-\mu)^{2}}{2 \sigma_{s}^{2}}} \cdot \sigma_{w}^{2}$ and $\sigma_{i}^{2}$ are the AWGN and IN variances which define the input SNR and SINR as SNR = $10 \log _{10}\left(\frac{\sigma_{\bar{x}}^{2}}{\sigma_{w}^{2}}\right)$ and SINR $=10 \log _{10}\left(\frac{\sigma_{\bar{x}}^{2}}{\sigma_{i}^{2}}\right)$, respectively, where $\sigma_{\bar{x}}^{2}$ is the variance of the transmitted signal.

Assuming perfect synchronization, the received signal can be expressed as

$$
r_{m}=\left\{\begin{array}{ll}
\bar{x}_{m}+w_{m}, & \mathcal{H}_{0} \\
\bar{x}_{m}+w_{m}+i_{m}, & \mathcal{H}_{1}
\end{array} \quad m=0,1, \ldots, M-1\right.
$$

$\bar{x}_{m}, w_{m}$ and $i_{m}$ are assumed to be mutually independent. The null hypothesis $\mathcal{H}_{0}$ implies the absence of IN, $P\left(\mathcal{H}_{0}\right)=$ $(1-p)$, whereas the alternative hypothesis $\mathcal{H}_{1}$ implies the presence of IN, $P\left(\mathcal{H}_{1}\right)=p$. In order to reduce the energy of IN, a blanking, clipping or hybrid nonlinear preprocessor is applied at the front-end of the receiver (before the SCFDMA demodulator) as illustrated in Fig. 1. The output of these devices are

- Blanking

$$
y_{m}=\left\{\begin{array}{ll}
r_{m}, & \left|r_{m}\right| \leq T_{b} \\
0, & \left|r_{m}\right|>T_{b}
\end{array} \quad m=0,1, \ldots, M-1\right.
$$

where $T_{b}$ is the blanking threshold.

- Clipping

$$
y_{m}=\left\{\begin{array}{ll}
r_{m}, & \left|r_{m}\right| \leq T_{c} \\
T_{c} e^{j \arg \left(r_{m}\right)}, & \left|r_{m}\right|>T_{c}
\end{array} \quad m=0,1, \ldots, M-1\right.
$$

where $T_{c}$ is the clipping threshold.
- Hybrid

$$
y_{m}= \begin{cases}r_{m}, & \left|r_{m}\right| \leq T_{c} \\ T_{c} e^{j \arg \left(r_{m}\right)}, & T_{c}<\left|r_{m}\right| \leq T_{b} \\ 0, & \left|r_{m}\right|>T_{b}\end{cases}
$$

where $r_{k}$ and $y_{k}$ are the input and output of the nonlinear device, respectively. It is noted that the device only processes the amplitude of the received signal without modifying its phase. The selection of the threshold(s) is the key for achieving best performance. For instance, if the threshold(s) is too small, many unaffected samples of the useful transmitted signal will be blanked/clipped resulting in poor bit error rate performance. On the other hand, for very large threshold(s), IN will be overlooked and will become part of the detected signal hence will degrade performance. In [11], a theoretical expression for the output SNR was derived as

$$
\mathrm{SNR}_{O F D M A}=\frac{2 K_{o}^{2}}{E_{\text {out }}-2 K_{o}^{2}}
$$

where $K_{o}$ is a real constant and $E_{\text {out }}$ is the total signal power at the output of the nonlinear preprocessor. These parameters are derived in [11] for the blanking, clipping and hybrid methods. These expressions will be used to verify the accuracy of our simulation model.

After the nonlinear device, the received signal $y_{m}$ is passed through the $M$-point DFT to produce $\left\{Y_{l}: l=0,1, \ldots M-1\right\}$ and the corresponding signal after the subcarrier demapping device is $\left\{\bar{Y}_{m}: m=0,1, \ldots N-1\right\}$. Finally, the signal after the $N$-point IDFT is given as $\left\{\bar{y}_{n}: n=0,1, \ldots, N-1\right\}$.

\section{THE RELATIONSHIP BETWEEN PAPR AND THRESHOLD $(S)$}

In this section we discuss the impact of PAPR on the blanking/clipping threshold. It is intuitive to think that if the average PAPR of the transmitted signal symbols is reduced, then this will make IN more distinguishable from the useful transmitted signal and therefore can improve the blanking/clipping process at the receiver. One way of accomplishing this is by using an SC-FDMA system such as LFDMA and IFDMA schemes.

For further clarity, an illustrative example is presented in Fig. 3 showing plots of OFDMA, LFDMA and IFDMA signals in addition to IN pulses. Depending on the modulation scheme applied, this figure presents three different scenarios. First, in case of the OFDMA system it can be seen that when the threshold $T_{\text {OFDMA }}$ is considered, only two IN pulses will be recognized $\{$ IN5, IN6 $\}$; whereas if $T_{\text {LFDMA }}$ or $T_{\text {IFDMA }}$ is used, this will allow detecting more IN pulses, however, unaffected samples will also trigger the nonlinear device causing IN detection errors. The second scenario is considering the LFDMA system with blanking threshold $T_{\text {LFDMA }}$ in which the preprocessor will be able to identify more IN pulses $\{$ IN1, IN4, IN5, IN6 $\}$. The third scenario is when IFDMA is adopted which allows using $T_{\text {IFDMA }}$ with zero IN detection errors in addition to eliminating all the IN pulses.

From Fig. 3, the amount of reduction in blanking/clipping threshold is referred to as threshold gain $\left(\mathrm{TG}_{\mathrm{L} / \mathrm{I}}=T_{\mathrm{LFDMA} / \mathrm{IFDMA}}-T_{\text {OFDMA }}\right)$. It will be shown later that the higher the TG, the more performance enhancement is 


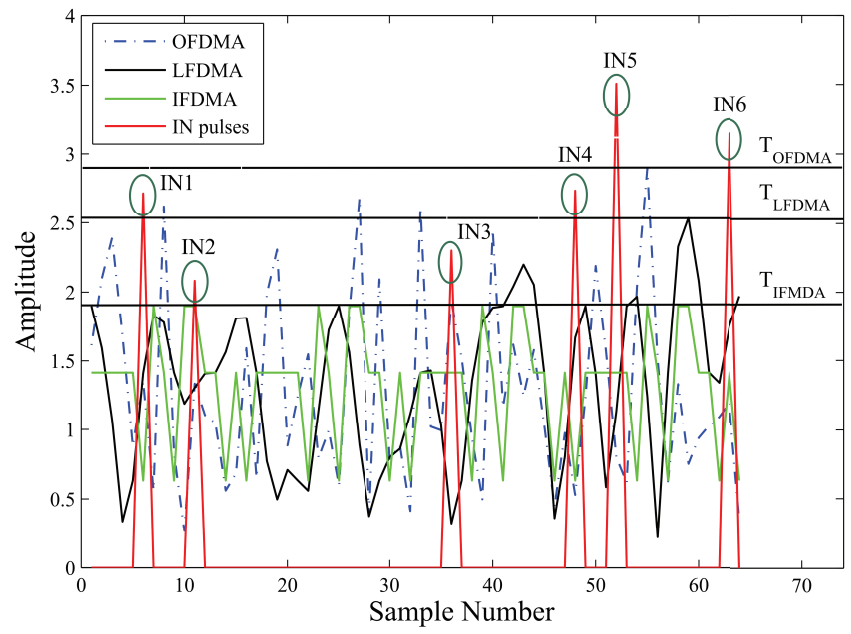

Fig. 3: An example of blanking/clipping thresholds for OFDMA, LFDMA and IFDMA when $N=16, M=64, p=0.1$ and SINR $=-6 \mathrm{~dB}$.

achieved in terms of the output SNR. For better realization of the proposed system, it is important to investigate the PAPR distribution of OFDMA, LFDMA and IFDMA systems. Therefore, we introduce the complementary cumulative distribution function $(\mathrm{CCDF})$ of the PAPR of these systems.

\section{A. OFDMA System}

The CCDF of PAPR is defined as the probability that the PAPR of a data block exceeds a given threshold $\left(\mathrm{PAPR}_{o}\right)$. A simple expression of the CCDF in the case of OFDMA system is derived in [15] and can be rewritten in terms of peaks $(P)$ instead of PAPR as

$$
C C D F=1-\operatorname{Pr}\left(P \leq P_{o}\right)=1-\left(1-e^{\left(-P_{o}\right)}\right)^{N}
$$

\section{B. LFDMA system}

In LFDMA, the time domain signal is obtained by substituting (2) into (4) and by letting $m=Q n+q$, where $\{n=0,1, \ldots, N-1\}$ and $\{q=0,1, \ldots, Q-1\}$, the LFDMA transmitted signal can be expressed as [16]

$$
\bar{x}_{n Q+q}= \begin{cases}\frac{1}{Q} x_{n}, & q=0 \\ \frac{\left(1-e^{j 2 \pi \frac{q}{Q}}\right)}{Q M} \sum_{p=0}^{M-1} \frac{x_{p}}{1-e^{j 2 \pi\left\{\frac{(n-p)}{M}+\frac{q}{N Q}\right\}},} & q \neq 0\end{cases}
$$

From (16), it is clear that the LFDMA transmitted signal has copies of the original sequence in $\{q=0\}$ positions with scaling factor of $1 / Q$; whereas in $q \neq 0$ positions values of the sum of all the input sequences in the input block with different complex scaling factors. This, however, increases the PAPR of the LFDMA signal.

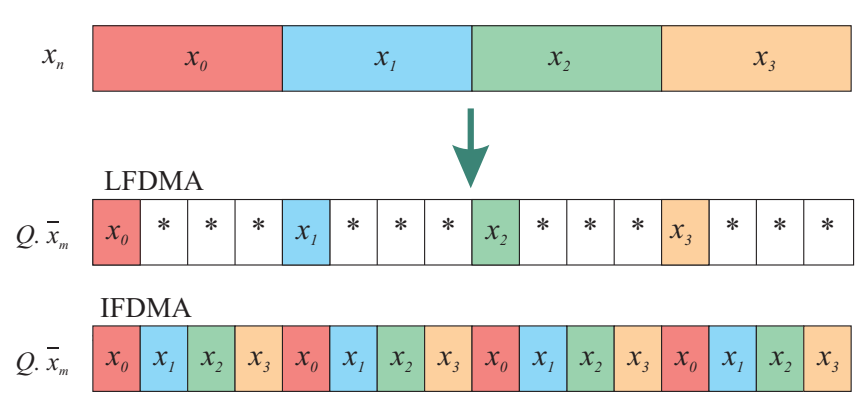

Fig. 4: Time domain symbols of LFDMA and IFDMA subcarrier mapping modes when $N=4, Q=4$ and $M=16$.

\section{IFDMA system}

Similarly, the IFDMA transmitted signal is obtained by substituting (3) into (4). Let $m=N q+n$, where $\{n=0,1, \ldots, N-1\}$ and $\{q=0,1, \ldots, Q-1\}$, then

$$
\bar{x}_{m}\left(=\bar{x}_{N q+n}\right)=\frac{1}{Q} x_{n}
$$

It is interesting to notice from this expression that the IFDMA time domain symbols are a repetition of the input symbols $x_{n}$. This implies that the PAPR of the IFDMA signal is equal to the PAPR of the single-carrier signal. For better illustration, Fig. 4 depicts a schematic diagram of time domain signals for LFDMA and IFDMA subcarrier mapping schemes.

To compare the PAPRs of OFDMA, LFDMA and IFDMA, a MATLAB simulation is conducted in which 100000 random symbols are generated and the corresponding symbol peaks are calculated. Fig. 5 shows the CCDF of peaks for the three systems when 16QAM symbol constellations is used. These results are obtained for a total number of subcarriers $(M=64)$, input symbols $(N=16)$ and spreading factor $(Q=4)$. It can be seen that in the case of OFDMA the analytical results, obtained from (15), correlate well with the simulation ones. It is also observed that IFDMA consistently provides the lowest peaks with about 3.5 unites peak reduction relative to OFDMA at CCDF of $10^{-4}$ whereas about 1.5 unites of gain is obtained by LFDMA at the same CCDF value. Furthermore, it is important to point out that IFDMA has two unique properties. First and as anticipated above (17), IFDMA and single-carrier systems have exact peak distribution. The second property is the sharp drop in CCDF at around 1.9. In general, the reduction in the peak values obtained by SC-FDMA means that more of the transmitted signal energy is contained close to the average value and hence larger TG value can be attained.

\section{The Probability of IN Detection ERror}

The probability of IN detection error $\left(P_{e}\right)$ is defined as the probability that the amplitude of the received sample, $A_{r}=\left|r_{m}\right|$, exceeds the blanking/clipping threshold when it is not affected by IN and it is mathematically expressed as $P_{e}=P\left(B, \mathcal{H}_{0}\right)$, where $B$ is the event of blanking/clipping the received signal exceeding $T$. According to Bayes' theorem, $P\left(B, \mathcal{H}_{0}\right)=P\left(B \mid \mathcal{H}_{0}\right) P\left(\mathcal{H}_{0}\right)$. Thus,

$$
P_{e}=P\left(A_{r}>T \mid \mathcal{H}_{0}\right) P\left(\mathcal{H}_{0}\right)
$$


For the OFDMA system, in the absence of IN the amplitude of the received signal has Rayleigh distribution with parameter $\sigma^{2}=\sigma_{\bar{x}}^{2}+\sigma_{w}^{2}$ and its conditional PDF can be expressed as

$$
f_{A_{r}}\left(r \mid \mathcal{H}_{0}\right)=\frac{r}{\left(\sigma_{\bar{x}}^{2}+\sigma_{w}^{2}\right)} e^{-\left(\frac{r^{2}}{2\left(\sigma_{\bar{x}}^{2}+\sigma_{w}^{2}\right)}\right)} P\left(\mathcal{H}_{0}\right)
$$

and the probability of IN detection error is

$$
\begin{aligned}
P_{e} & =\int_{T}^{\infty} f_{A_{r}}\left(r \mid \mathcal{H}_{0}\right) \mathrm{d} r \\
& =e^{-\frac{T^{2}}{2\left(\sigma_{\bar{x}}^{2}+\sigma_{w}^{2}\right)}}(1-p)
\end{aligned}
$$

Some numerical results of (20) are shown in Fig. 6 along with simulation results for OFDMA, LFDMA and IFDMA. It is clearly observed that the analytical and simulation results of the OFDMA are matching. Our results from this point onward are based on an OFDM system consisting of $M=64$ subcarriers, $N=16, Q=4$, 16QAM modulation, $\sigma_{\bar{x}}^{2}=(1 / 2) E\left[\left|\bar{x}_{m}\right|^{2}\right]=$ $1, \sigma_{w}^{2}=(1 / 2) E\left[\left|w_{m}\right|^{2}\right]$ and $\sigma_{i}^{2}=(1 / 2) E\left[\left|i_{m}\right|^{2}\right]$.

In general, it is evident that both LFDMA and IFDMA exhibit lower probability of IN detection error in comparison with OFDMA. The behavior of the probability can be divided into two regions. The first region is $\{T \lesssim 2\}$ during which SC-FDMA systems do not provide any reduction. It can also be extracted from this figure that at $\{T \simeq 2\}$ about $\{\simeq 10 \%\}$ of the signal samples will exceed this threshold irrespective of the system being used. In the second region $\{T \gtrsim 2\}$ it becomes noticeable that both SC-FDMA schemes have lower probability of IN detection error whilst IFDMA provides the lowest probability. The general trend in this region is that as $T$ increases the probability is minimized. For instance, in the LFDMA system and at $T=2.5$, the probability is reduced by about 0.3 order of magnitude; whereas at $T=3$ the probability is minimized by about 1 order of magnitude. However, in the case of IFDMA it is interesting to note that at about $T=2$, the probability falls dramatically offering zero blanking error beyond this threshold. The reduction in the probability implies that the SNR performance will improve as will be further discussed in the next section.

\section{The OutPut SNR Versus THRESHOLD}

In this section we analyze the performance of the proposed system, by means of simulation, in terms of SNR at the output of the three nonlinear devices which is calculated as

$$
\operatorname{SNR}_{O / L / I F D M A}=\frac{E\left[\left|\bar{x}_{m}\right|^{2}\right]}{E\left[\left|y_{m}-\bar{x}_{m}\right|^{2}\right]}
$$

The output SNRs for OFDMA, LFDMA and IFDMA as a function of blanking, clipping and hybrid threshold are illustrated in Fig. 7(a), (b) and (c), respectively, when SINR = $-10 \mathrm{~dB}, p=0.01$ and input $\mathrm{SNR}=30 \mathrm{~dB}$. From this figure it can be seen that the analytical results of OFDMA system for the three nonlinear devices, obtained from (14), are in good agreement with the simulation ones and this verifies the accuracy of our simulations. As anticipated, we can see that LFDMA performs better than OFDMA whereas IFDMA

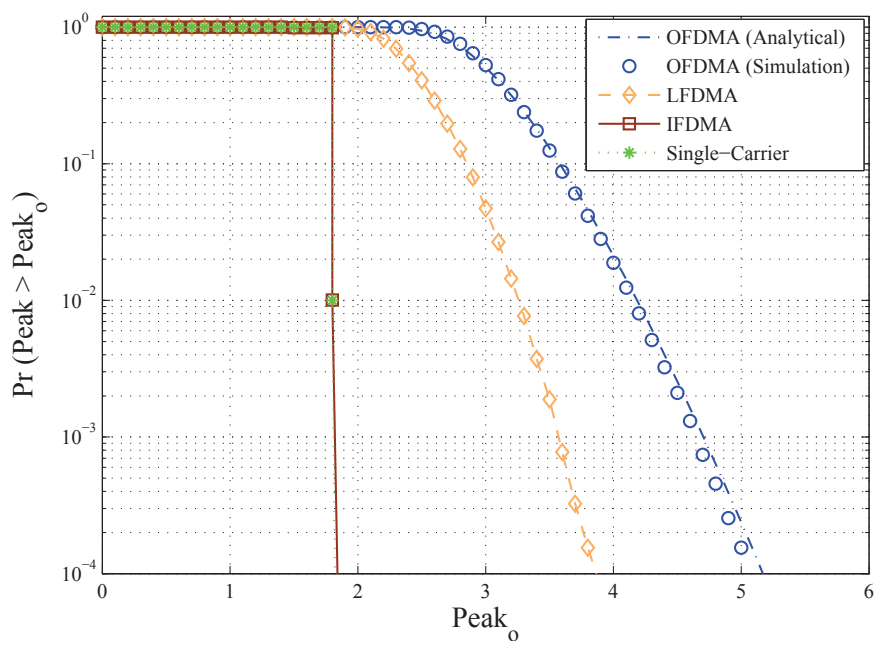

Fig. 5: CCDF for OFDMA, LFDMA and IFDMA when $M=64, N=16$ and $Q=4$.

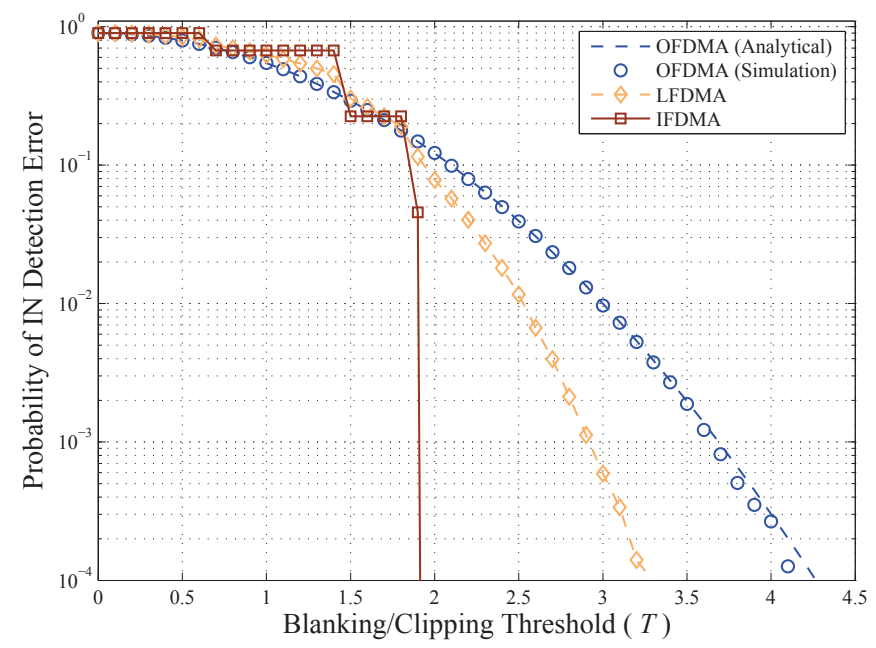

Fig. 6: Probability of IN detection error versus blanking/clipping threshold for OFDMA, LFDMA and IFDMA systems.

outperforms both systems. There is a general trend, for all systems under study, that when $T$ is too small $\{T \lesssim 2\}$ the system performance degrades significantly since a great amount of the useful signal energy is lost. On the other hand, if $T$ is too high $\{T \rightarrow \infty\}$ no blanking/clipping takes place which allows all the IN energy to be part of the detected signal. In such a scenario, the output SNR approaches $10 \mathrm{~dB}$ as can clearly be observed from this figure and it can be mathematically expressed as

$$
\operatorname{SNR}_{O / L / I F D M A}(T \rightarrow \infty)=10 \log _{10}\left(\frac{\sigma_{\bar{x}}^{2}}{\sigma_{w}^{2}+p \sigma_{i}^{2}}\right)
$$

Also, when $p \sigma_{i}^{2} \gg \sigma_{w}^{2}$, (22) can be approximated to $\simeq$ $10 \log _{10}\left(\frac{1}{p \sigma_{i}^{2}}\right)$. It is interesting to see that for each system there exists an optimal blanking/clipping/hybrid threshold that maximizes the output SNR. Furthermore, it is evident that LFDMA based scheme can provide gains in the output SNR of up to $2 \mathrm{~dB}, 1 \mathrm{~dB}$ and $2 \mathrm{~dB}$ for blanking, clipping and hybrid, respectively; whereas the IFDMA offers SNR improvement 
of up to $4 \mathrm{~dB}, 3 \mathrm{~dB}$ and $3 \mathrm{~dB}$, respectively. In addition, it is interesting to note from Fig. 7(a) that the performance of IFDMA sharply improves at blanking threshold of about 2 whereas in the other systems the improvement is gradual.

\section{CONCLUSION}

In this paper we have investigated the performance of SCFDMA with blanking, clipping and hybrid devices at the receiver in the presence of IN. The results clearly show that the proposed technique is superior over the conventional OFDMAbased systems in the form of minimized probability of IN detection error and an increase in the output SNR which can be up to $4 \mathrm{~dB}, 3 \mathrm{~dB}$ and $3 \mathrm{~dB}$ when blanking, clipping and hybrid schemes are applied, respectively.

\section{REFERENCES}

[1] X. Cheng, R. Cao, and L. Yang, "Relay-aided amplify-and-forward powerline communications," IEEE Trans. Smart Grid, vol. PP, no. 99, pp. $1-8,2013$.

[2] M. Zimmermann and K. Dostert, "A multipath model for the powerline channel,” IEEE Trans. Commun., vol. 50, no. 4, pp. 553-559, 2002.

[3] _ - "Analysis and modeling of impulsive noise in broad-band powerline communications," IEEE Trans. Electromagn. Compat., vol. 44, pp. 249258, Feb. 2002.

[4] D. Anastasiadou and T. Antonakopoulos, "Multipath characterization of indoor power-line networks," IEEE Trans. Power Del., vol. 20, no. 1, pp. 90-99, Jan. 2005.

[5] H. Meng, Y. L. Guan, and S. Chen, "Modeling and analysis of noise effects on broadband power-line communications," IEEE Trans. Power Del., vol. 20, no. 2, pp. 630-637, Apr. 2005.

[6] P. Cuntic and A. Baiant, "Analysis of modulation methods for data communications over the low-voltage grid," Proceeding of the 7th International Conference on Telecommunication ConTEL, pp. 20-23, 2002.

[7] Y. H. Ma, P. L. So, and E. Gunawan, "Performance analysis of OFDM system for broadband power line communications under impulsive noise and multipath effects," IEEE Trans. Power Del., vol. 20, no. 2, pp. 674682, Apr. 2005.

[8] M. Gotz, M. Rapp, and K. Dostert, "Power line channel characteristics and their effect on communication system design," IEEE Commun. Mag., vol. 42, no. 4, pp. 78-86, 2004.

[9] M. Ghosh, "Analysis of the effect of impulse noise on multicarrier and single carrier QAM systems," IEEE Trans. Commun., vol. 44, no. 2, pp. 145-147, Feb. 1996

[10] S. V. Zhidkov, "Performance analysis and optimization of OFDM receiver with blanking nonlinearity in impulsive noise environment," IEEE Trans. Veh. Technol., vol. 55, no. 1, pp. 234-242, Jan. 2006.

[11] _ "Analysis and comparison of several simple impulsive noise mitigation schemes for ofdm receivers," IEEE Trans. commun., vol. 56, no. 1, pp. 5-9, Jan 2008.

[12] E. Alsusa and K. Rabie, "Dynamic peak based threshold estimation method for mitigating impulsive noise in power-line communication systems," IEEE Trans. Power Del., no. 99, pp. 1-8, 2013.

[13] K. Rabie and E. Alsusa, "Preprocessing based impulsive noise reduction for powerline communications," to appear in IEEE Trans. Power Del., 2014.

[14] H. Myung, J. Lim, and D. Goodman, "Single carrier FDMA for uplink wireless transmission," IEEE Veh. Technol. Mag., vol. 1, no. 3, pp. 30-38, 2006.

[15] R. van Nee and R. Prasad, "OFDM for wireless multimedia communications," Artech House, 2000.

[16] H. Myung, J. Lim, and D. Goodman, "Peak-to-average power ratio of single carrier FDMA signals with pulse shaping," in Proc. IEEE PIMRC'06, pp. 1-5, Sep. 2006.

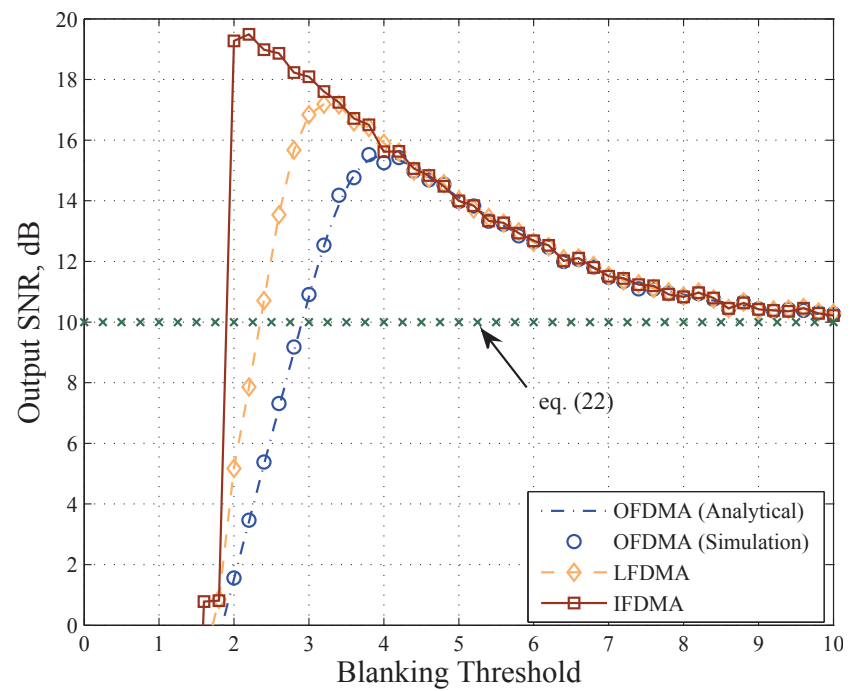

(a) Blanking.

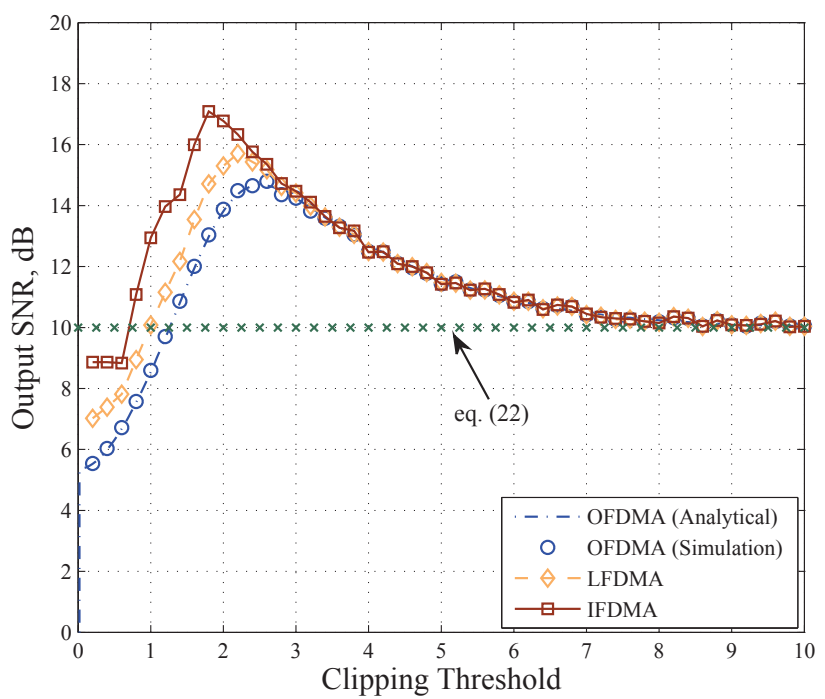

(b) Clipping.

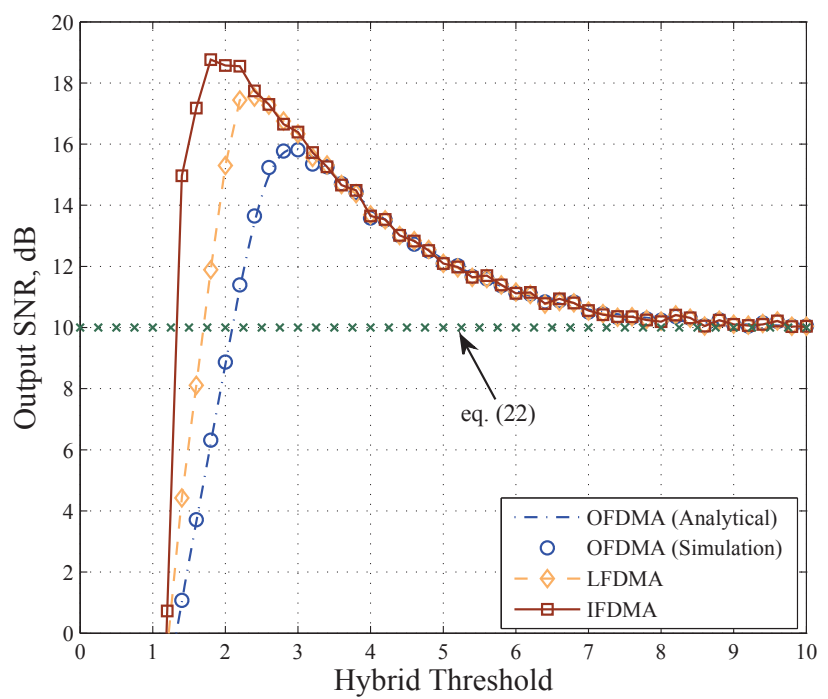

(c) Hybrid $\left(T_{1}=1.4 T_{2}\right)$.

Figure 7: The output SNR versus blanking/clipping/hybrid threshold for OFDMA, LFDMA and IFDMA systems when SINR $=-10 \mathrm{~dB}, p=0.01$ and $\mathrm{SNR}=30 \mathrm{~dB}$. 